\title{
ONE-TO-ONE OPERATORS ON FUNCTION SPACES
}

\author{
STEPHEN T. L. CHOY
}

\begin{abstract}
For a Banach algebra $A$ one-to-one operators with closed range on $C_{0}(S, A)$ are characterized in terms of the associated vector measures given by the Riesz Representation Theorems. Multiplicatively symmetric operators are also studied.
\end{abstract}

1. Introduction. Let $S$ be a locally compact Hausdorff topological space and let $A$, $B$ be Banach algebras. Denote by $C_{0}(S, A)$ the algebra of continuous functions from $S$ to $A$ vanishing at infinity endowed with the uniform norm. If $A=\mathrm{C}$, the set of all complex numbers, we simply write $C_{0}(S)$. Let $C_{0}(S) \hat{\otimes} A$ be the completion of the algebraic tensor product $C_{0}(S) \otimes A$ with respect to the least cross norm. Then $C_{0}(S, A)=C_{0}(S) \hat{\otimes} A$, where the equation indicates isometry between the two spaces.

Continuous linear operators $T: C_{0}(S, A) \rightarrow B$ are represented by measures $m$ : 仍 $(S) \rightarrow E^{[}\left[A, B^{* *}\right]$ in, for example, Brooks and Lewis [4] and Batt and Berg [3]; where $\operatorname{li}(S)$ is the $\sigma$-algebra of Borel subsets of $S$ and $B^{* *}$ is the second dual of $B$. Mapping properties of representing measures are studied, for example, by Bilyeu and Lewis [2], Brooks and Lewis [4], Johnson [9] and the author [5].

A bounded linear operator $T: C_{0}(S, A) \rightarrow C_{0}(S, A)$ is called a multiplicatively symmetric operator if $T(f T(g))=T(T(f) g)$ for all $f, g \in C_{0}(S, A)$. Dhombres [6] showed that multiplicatively symmetric operators coincide with exaves for the case when $S$ is compact, $A=\mathrm{C}$ and $\|T\|=T(1)=1$. In $\$ 2$ we characterize one-to-one operators with closed range and multiplicatively symmetric operators which are one-to-one in terms of their representing measures. An example is given in $\S 3$ to explain Theorem 2.1. More interesting examples of representing measures may be found, for example, in Brooks and Lewis [4].

Throughout this paper multiplication in the second dual of Banach algebras is defined by the left Arens product. Duncan and Hosseiniun [8] is a convenient reference for the Arens product.

2. One-to-one operators. For $f^{\prime} \in C_{0}^{*}(S, A), x \in A$, there is a unique regular Borel measure $\mu\left(x, f^{\prime}\right)$ such that $\int f d \mu\left(x, f^{\prime}\right)=f^{\prime}(f \cdot x)$ for $f \in C_{0}(S)$. Therefore, for $e \in \mathscr{B}(S), x \in A, 1_{e} \otimes x$ can be viewed as an element of $C_{0}^{* *}(S, A)$ defined by $\left(1_{e} \otimes x\right)\left(f^{\prime}\right)=\mu\left(x, f^{\prime}\right)(e)$. Recall that $T^{* *}\left(1_{e} \otimes x\right)=m(e) x$. Let $\mathscr{P}(S)$ be the class of all Borel partitions of $S$.

Received by the editors April 14, 1982.

1980 Mathematics Subject Classification. Primary 47B38: Secondary 46G10.

Key words and phrases. Vector-valued measures, representing measures, one-to-one operators, multiplicatively symmetric operators, weakly compact operators, Arens product. 
TheOREM 2.1. An operator $T: C_{0}(S, A) \rightarrow C_{0}(S, A)$ is one-to-one and has a closed range iff $\sum_{i=1}^{n} m\left(e_{i}\right) x_{i}=0$ implies that $x_{i}=0$ for $\left\{e_{i}\right\} \in \mathscr{9}(S)$ and $x_{i} \in A$ with $\left\|x_{i}\right\| \leqslant 1(i=1,2, \ldots, n)$.

Proof. Suppose, for every $\sum_{i=1}^{n}\left(1_{e_{1}} \otimes x_{i}\right), T^{* *}\left(\sum 1_{e_{1}} \otimes x_{i}\right)=0$ implies $\sum\left(1_{e_{1}} \otimes x_{i}\right)$ $=0$. Let $g_{0}^{\prime}$ be any element in $C_{0}^{*}(S, A)$ and let

$$
M=\left\{T^{* *}\left(\sum 1_{e}, \otimes x_{i}\right):\left\{e_{i}\right\} \in \mathcal{Q P}(S), x_{i} \in A,\left\|x_{i}\right\| \leqslant 1\right\} .
$$

Then $M$ is a linear subspace of $C_{0}^{* *}(S, A)$. Define a linear functional $f_{0}^{\prime}$ on $M$ by

$$
f_{0}^{\prime}\left(T^{* *}\left(\sum 1_{e_{1}} \otimes x_{i}\right)\right)=\left(\sum 1_{e_{i}} \otimes x_{i}\right)\left(g_{0}^{\prime}\right)
$$

Since $T^{* *}\left(\sum 1_{e_{1}} \otimes x_{i}\right)=0$ implies $\Sigma 1_{e_{1}} \otimes x_{i}=0, f_{0}^{\prime}$ is well defined. Furthermore,

$$
\left|f_{0}^{\prime}\left(T^{* *}\left(\Sigma 1_{e_{1}} \otimes x_{i}\right)\right)\right|=\left|\left(\Sigma 1_{e_{1}} \otimes x_{i}\right)\left(g_{0}^{\prime}\right)\right| \leqslant\left\|g_{0}^{\prime}\right\| \text {. }
$$

Therefore $f_{0}^{\prime}$ is continuous on $M$ and so can be extendedto a continuous linear functional $f^{\prime}$ on $C_{0}^{* *}(S, A)$. Since

$$
f^{\prime}\left(T^{* *}\left(\sum 1_{e_{1}} \otimes x_{i}\right)\right)=\left(\sum 1_{e_{1}} \otimes x_{i}\right)\left(g_{0}^{\prime}\right),
$$

we see, by taking the limit process, that

$$
f^{\prime}\left(T^{* *}(f)\right)=f\left(g_{0}^{\prime}\right) \quad\left(f \in C_{0}(S, A)\right) .
$$

Recall that $T^{* *}(f) \in C_{0}(S, A)$ for all $f \in C_{0}(S, A)$. Hence, when $f^{\prime}$ is considered as a functional on $C_{0}(S, A), g_{0}^{\prime}=T^{*} f^{\prime}$ and so $T^{*}$ is an onto mapping. Therefore $T$ is a one-to-one operator with closed range [10, Theorem 4.14].

Conversely, suppose $T$ is one-to-one with closed range and $\Sigma m\left(e_{1}\right) x_{1}=0$. Then $T^{*}$ is onto [10, Corollary of 4.12] and, for all $f^{\prime} \in C_{0}^{*}(S, A), T^{* *}\left(\Sigma 1_{e}, \otimes x_{i}\right)\left(f^{\prime}\right)=0$. Let $g^{\prime} \in C_{0}^{*}(S, A)$. Then there is $f^{\prime} \in C_{0}^{*}(S, A)$ such that $g^{\prime}=T^{*} f^{\prime}$. Hence

$$
\left(\Sigma 1_{e_{1}} \otimes x_{i}\right)\left(g^{\prime}\right)=\left(\Sigma 1_{e_{1}} \otimes x_{i}\right)\left(T^{*} f^{\prime}\right)=T^{* *}\left(\sum 1_{e_{1}} \otimes x_{i}\right)\left(f^{\prime}\right)=0 .
$$

That is $\Sigma\left(1_{e_{1}} \otimes x_{i}\right)=0$ in $C_{0}^{* *}(S, A)$ and so $x_{i}=0$ for $i=1, \ldots, n[4$, Lemma 2.1].

It is easy to verify by an argument similar to [5, Proposition 2.2] that $T$ : $C_{0}(S, A) \rightarrow C_{0}(S, A)$ is a multiplier iff $T^{* *}: C_{0}^{* *}(S, A) \rightarrow C_{0}^{* *}(S, A)$ is a multiplier. Therefore $T$ is a multiplier iff

$$
\left(1_{e_{1}} \otimes x\right)\left(m\left(e_{2}\right) y\right)=\left(m\left(e_{1}\right) x\right)\left(1_{e_{2}} \otimes y\right)
$$

for all $e_{1}, e_{2} \in \Re(S), x, y \in A$. The following theorem shows the difference between multipliers and multiplicatively symmetric operators. Brooks and Lewis defined supports, supp $m$, of weakly regular measures $m$ and showed that $\operatorname{supp} m=\operatorname{supp} T$ in [4].

THEOREM 2.2. A one-to-one operator $T$ is multiplicatively symmetric iff

$$
\left(1_{e_{1}} \otimes x\right)\left(m\left(e_{2}\right) y\right)=\left(m\left(e_{1}\right) x\right)\left(1_{e_{2}} \otimes y\right)
$$

for all $e_{1}, e_{2} \in \mathscr{B}(\operatorname{supp} m)$ and $x, y \in A$.

Proof. Suppose $T$ is a one-to-one multiplicatively symmetric operator. Then

$$
T(f T(g))=T(T(f) g) \quad\left(f, g \in C_{0}(S, A)\right) .
$$


Therefore $f T(g)=T(f) g$ for all $f, g \in C_{0}(S, A)$. Hence, by an argument similar to [5, Proposition 2.1], $F T^{\prime \prime}(G)=T^{\prime \prime}(F) G$ for all $F, G \in C_{0}^{* *}(S, A)$. In particular

$$
\left(1_{e_{1}} \otimes x\right)\left(m\left(e_{2}\right) y\right)=\left(m\left(e_{1}\right) x\right)\left(1_{e_{2}} \otimes y\right)
$$

for all $e_{1}, e_{2} \in \Re(\operatorname{supp} m)$ and $x, y \in A$.

Conversely, suppose $\left(1_{e_{1}} \otimes x\right)\left(m\left(e_{2}\right) y\right)=\left(m\left(e_{1}\right) x\right)\left(1_{e_{2}} \otimes y\right)$ for $e_{1}, e_{2} \in$ $\Re(\operatorname{supp} m)$ and $x, y \in A$. Then

$$
\left(\sum_{i=1}^{n} 1_{e_{i}} \otimes x_{i}\right)\left(m\left(e_{j}\right) y_{j}\right)=\left(\sum_{i=1}^{n} m\left(e_{i}\right) x_{i}\right)\left(1_{e_{j}} \otimes y_{j}\right)
$$

for $e_{i}, e_{j} \in \mathscr{B}(\operatorname{supp} m), x_{i}, x_{j} \in A$. Since $\sum 1_{e_{1}} \otimes x_{i} \in C_{0}^{* *}(S, A)$ for $e_{i} \in \Re(\operatorname{supp} m)$ and $x_{i} \in A$, each $f \in C_{0}$ (supp $m, A$ ) can be considered as an element in $C_{0}^{* *}(S, A)$. Using Bartle's Bounded Convergence Theorem, we see

$$
f\left(m\left(e_{j}\right) y_{j}\right)=T^{* *}(f)\left(1_{e} \otimes y_{j}\right)
$$

and similarly $f\left(T^{* *}(g)\right)=T^{* *}(f) g$ for $f, g \in C_{0}(\operatorname{supp} m, A)$. For each $f \in C_{0}(S, A)$, the restriction of $f$ to $\operatorname{supp} m, f_{m}$, is in $C_{0}(\operatorname{supp} m, A)$. Since $T(f)=\int f_{m} d m=$ $T^{* *}\left(f_{m}\right)$, we have $T^{* *}\left(f_{m}\right) \in C_{0}(S, A)$ for every $f \in C_{0}(S, A)$. Therefore for $f$. $g \in C_{0}(S, A)$,

$$
\begin{aligned}
T(f T(g)) & =\int f T(G) d m=\int f_{m} T^{* *}\left(g_{m}\right) d m \\
& =\int T^{* *}\left(f_{m}\right) g_{m} d m=\int T(f) g d m \\
& =T(T(f) g) .
\end{aligned}
$$

This completes the proof of the theorem.

3. Remarks. Theorem 2.1 is valid when $A$ is only a Banach space. In the proof of sufficiency of Theorem $2.2, T$ is not necessarily to be one-to-one. It is interesting to determine whether Theorem 2.2 is true in general even if $T$ is not one-to-one.

4. Example. The paper concludes with an example to illustrate Theorem 2.1. Let $S$ be the natural numbers equipped with the discrete topology, and let $A$ be a Banach space. Denote

$$
1_{1}(A)=\left\{x=\left(x_{i}\right): x_{i} \in A \text { and } \sum_{n=1}^{\infty}\left\|x_{i}\right\|<\infty\right\} .
$$

Then $l_{1}(A)$ is a Banach space with the norm defined by $\|x\|=\Sigma\left\|x_{1}\right\|$ for $x=\left(x_{1}\right)$. It is shown by Dobrakov [7] that $C_{0}^{*}(S, A)=1_{1}\left(A^{*}\right)$.

EXAMPLE 4.1. Let $H$ be a Hilbert space, $\left\{\alpha_{n}\right\}$ be a sequence of complex numbers converging to zero and $\left|\alpha_{n}\right| \leqslant 1$. Define $T: C_{0}(S, H) \rightarrow C_{0}(S, H)$ by

$$
T f=\left(\alpha_{i} f_{i}\right) \quad\left(f=\left(f_{1}\right) \in C_{0}(S, H)\right) .
$$

Then the representing measure $m$ of $T$ is defined by $m(E)=\sum_{n \in E} \alpha_{n} e^{n}$, where $e^{n}=\left(e_{1}^{n}\right)$ with $e_{1}^{n}=0$ if $i \neq n$ and $e_{n}^{n}=1$. Then $m:$ of $(S) \rightarrow \mathcal{E}\left[H, C_{0}(S, H)\right]$ and 
$m(E) x=\left(\sum_{n \in E} \alpha_{n} e^{n}\right) x$. Set

$$
K=\left\{\sum_{i=1}^{n} m\left(E_{l}\right) x_{i}:\left\{E_{l}\right\} \in \mathbb{P}(S), x_{1} \in H \text { and }\left\|x_{i}\right\| \leqslant 1\right\} .
$$

Then $K \subset C_{0}(S, H)$. We shall show that $K$ is weakly conditionally compact. Recall that a set in a Hilbert space is relatively weakly sequentially compact iff it is bounded. Let $\left\{\gamma^{n}\right\}=\left\{\sum_{i=1}^{N_{n}} m\left(E_{1}^{n}\right) x_{i}^{n}\right\}$ be in $K$. Then each $\gamma^{n}$ is of the form $\gamma^{n}=\left(\alpha_{i} x_{i}^{n}\right)$. For each $i,\left\{\alpha_{i} x_{i}^{n}: n=1,2, \ldots\right\}$ is bounded in $H$ and so, without loss of generality, we can assume that $\left\{\alpha_{i} x_{i}^{n}\right\} \rightarrow y_{i} \in H$ weakly. Hence, for each $f_{i} \in H$, $\left\langle\alpha_{i} x_{i}^{n}, f_{i}\right\rangle \rightarrow\left\langle y_{i}, f_{i}\right\rangle$. For each $F=\left(f_{i}\right) \in 1_{1}(H)=C_{0}^{*}(S, H)$, since

$$
\left|\sum_{i=1}^{\infty}\left\langle\alpha_{i} x_{i}^{n}, f_{i}\right\rangle-\sum_{i=1}^{N}\left\langle\alpha_{i} x_{i}^{n}, f_{i}\right\rangle\right| \leqslant \sum_{i=N+1}^{\infty}\left\|f_{i}\right\| .
$$

it is easily verified that

$$
F\left(\left(\alpha_{i} x_{i}^{n}\right)\right)=\sum_{i=1}^{\infty}\left\langle\alpha_{i} x_{i}^{n}, f_{i}\right\rangle \rightarrow \sum_{i=1}^{\infty}\left\langle y_{i}, f_{i}\right\rangle=F\left(\left(y_{i}\right)\right) .
$$

That is $K$ is weakly conditionally compact and we conclude that $T$ is weakly compact. Furthermore $T$ is one-to-one and onto iff $\alpha_{n} \neq 0$ for each $n$.

I would like to thank the referee for pointing out Dobrakov's result [7] to me. It shortens the proofs of Example 4.1 in the original version of the manuscript.

\section{REFERENCES}

1. R. G. Bartle, A general hilinear integral. Studia Math. 15 (1956). 337-352.

2. R. Bilveu and P. Lewis. Some mapping properties of representing measures. Ann. Mat. Pura Appl. 109 (1976), 273-287.

3. J. Batt and E. J. Berg. Linear hounded transformations on the space of comtinuous functhons. J. Functional Analysis 4 (1969). 215-239.

4. J. K. Brooks and P. W. Lewis, Linear operators and cector measures. Trans. Amer. Math. Soc. 192 (1974), 139-162.

5. S. T. L. Choy. Integral representation of multiplicative, imvolution presercing operators in L'( $\left.C_{0}(S, A), B\right)$, Proc. Amer. Math. Soc. 83 (1981), 54-58.

6. J. G. Dhombres, A functional characterization of marhoxian linear exates, Bull. Amer. Math. Soc. 81 (1975). 703-706.

7. I. Dobrakov, On representation of linear operators on $C_{0}(T . X)$. Czechoslovak Math. J. 21 (1971). $13-30$.

8. J. Duncan and S. A. R. Hosseiniun. The second dual of a Banach algehra. Proc. Roy. Soc. Edinburgh Sect. A 84 (1979), 309-325.

9. G. W. Johnson, Integral representation of multiplicatice. incolution preserving operators in L̇( (C $S) . E)$. Proc. Amer. Math. Soc. 23 (1969). 373-377.

10. W. Rudin. Functional analisis. Mc(jraw-Hill. New York. 1973.

Department of Mathematics. National University of Singapore. Republic of Singapore. (0511) 